\title{
Infestation expérimentale d'une Hélicelle par huit espèces de Trématodes digénétiques appartenant à quatre familles différentes (1)
}

\author{
par Jean TIMON-DAVID
}

On admet généralement que la spécificité parasitaire des Digènes vis-à-vis du premier hôte intermédiaire (Mollusque) est très stricte. C'est l'opinion que formule C. H. Wright (1960), qui écrit: "The evidence, however, is becoming increasingly strong in favour of a very hight degree of specificity in trematode-molluscs relationships. »Cette manière de voir n'est pas toujours confirmée par l'expérience et je désire synthétiser ici les résuitats des recherches que je poursuis depuis une dizaine d'années en réalisant des contaminations expérimentales du Pulmoné terrestre Helicella (Helicopsis) arenosa Ziegler (Rossmässler).

On sait que chez les Pulmonés aquatiques (Limnées, Planorbes, Physes, etc.), la spécificité parasitaire est en grande partie conditionnée par le mécanisme de l'attraction miracidienne qui préiude à la pénétration chez le Mollusque. Il n'en est pas de même chez les Pulmonés terrestres où la contamination est passive, résultant de l'ingestion des œufs qui accompagnent les aliments. Dans ce cas, ce sont uniquement les facteurs biochimiques du tube digestif qui contrôlent l'éclosion, la libération du miracidium et le développement du parasite $(\mathrm{pH}$, équipement enzymatique, présence possible d'anticorps, nature du métabolisme, etc.).

Dans l'étude expérimentale de la spécificité, l'utilisation des Mollusques terrestres présente un avantage très net par rapport aux Mollusques aquatiques: chez ces derniers, en effet, la contamination ne peut être «forcée » puisque nous ne disposons pas de moyens permettant d'agir sur l'attraction miracidienne. Au contraire, chez les Mollusques terrestres, il est facile d'intervenir en provoquant l'ingestion des œufs par la voie orale, et ce sont seulement les processus biochimiques mentionnés précédemment qui peuvent jouer le rôle de facteurs limitants. Les résultats des recherches que j'expose ici indiquent que ce contrôle paraît être assez lâche.

J'ai pu obtenir le développement expérimental chez cette Hélicelle, pour huit espèces de Trématodes Digènes, appartenant aux familles suivantes: Cyclocoelidae

(1) Communication présentée au $1^{\text {er }}$ Congrès international de Parasitologie, Rome, septembre 1964. 
(une espèce), Dicrocoeliidae (quatre espèces), Leucochloridiidae (deux espèces), Brachylaemidae (une espèce). Tous ces parasites ont pu être élevés au laboratoire, en grand nombre et en répétant plusieurs fois les contaminations. J'ai déjà décrit $(1957,1960)$ la technique très simple que j'ai mise au point pour réaliser ces contaminations massives. L'utilisation d'un seul repas infestant, réalisé à un moment exactement connu, permet de dater de façon très précise les diverses étapes du développement, l'âge de chaque forme larvaire étant ainsi parfaitement déterminé.

Il va de soi que des lots témoins recueillis le même jour et au même endroit ont toujours été examinés pour établir avec certitude l'absence d'infestation naturelle.

Je me propose d'examiner ici comparativement les résultats obtenus en soulignant les particularités des cycles respectifs et leur chronologie. Il y a de grandes différences dans la rapidité du développement chez les espèces étudiées. Les adultes qui ont fourni les œufs utilisés dans ces expériences sont des parasites de Reptiles, d'Oiseaux et de Mammifères dans le Sud-Est de la France (Bouches-du-Rhône), en particulier aux environs d'Aix-en-Provence (Le Tholonet). C'est de cette localité que proviennent les Hélicelles, ainsi que les Oiseaux et les Mammifères. Les Lézards qui ont fourni les Paradistomum ont été capturés sur l'île de Riou.

\section{$1^{\circ}$ Développement d'un Cyclocoelidé.}

J'ai obtenu pour la première fois le développement d'un Cyclocoelidé (Pseudhyptiasmus dollfusi T. D., des sacs aériens de Pica pica L.) chez un Mollusque terrestre. Dans les cas précédemment connus, c'étaient des Mollusques aquatiques qui étaient en cause: cycles de Tracheophilus sisowi Skrjabin, étudié par L. Szidat (1932), de Typhlocoelum cymbium (Diesing, 1850), étudié par H. W. Stunkard (1934), de Cyclocoelum microstomum (Creplin), étudié par T. A. Ginetsinskaia (1949), de Transcoelum oculeus (Kossack), étudié par V. Palm (1963). Dans tous ces cas, le Mollusque constitue l'hôte intermédiaire unique; les cercaires prennent naissance dans des rédies qu'elles quittent pour s'enkyster dans les tissus du voisinage.

En ce qui concerne $P$. dollfusi, les cercaires se développent aussi dans de grandes rédies, mais n'en sortent pas et s'enkystent sur place, de telle sorte que la rédie se transforme en un sac bourré de métacercaires. Certaines rédies de grande taille atteignent jusqu'à $4,5 \mathrm{~mm}$ et renferment de 130 à 150 métacercaires. La forme adulte a été facilement obtenue en faisant ingérer les Hélicelles contaminées par des Pigeons, des Corneilles noires et des Moineaux.

En dehors d'H. arenosa, la contamination expérimentale a été aussi réussie chez Leucochroa candidissima Drap., mais avec un taux d'infestation beaucoup plus faible $(10,7 \%$ au lieu de $80 \%)$. J'ajoute que j'ai trouvé à deux reprises dans la nature des infestations spontanées chez Zonites algirus L.

Voici quelques précisions concernant la durée du développement : 33 jours après le repas infestant, les Hélicelles renferment des rédies contenant des balles germinales ; 90 jours après le début de l'expérience, on trouve des rédies avec des cercaires bien développées; ces rédies se trouvent aussi. bien dans la masse viscérale que dans la 
cavité palléale ; il peut y en avoir plus de 50 par Mollusque. La forme adulte renfermant des œufs a été obtenue chez les Oiseaux 140 jours après l'infestation initiale du Mollusque.

\section{$2^{\circ}$ Développement de quatre espèces de Dicrocoeliidae.}

J'ai réussi à provoquer chez $H$. arenosa le développement expérimental de quatre espèces de cette famille :

A) Dicrocoelium lanceolatum (Rud.), la «petite Douve s classique des voies biliaires du mouton. Je me borne ici à indiquer que j'ai obtenu la formation des sporocystes et des cercaires (xiphidiocercaires à longue queue du type vitrina), sans insister davantage sur cette espèce, objet de si nombreux travaux. Le développement est lent : 123 jours pour des sporocystes avec balles germinales et jeunes cercaires; 186 jours pour les cercaires à queue bien développée.

B) Dicrocoelioides petiolatum (A. Railliet, 1900). L'adulte est fréquent dans la vésicule biliaire des Corvidés (Pie et Geai) en Provence; il a été aussi signalé chez le Moineau au Maroc et, avec doute, chez divers Passériformes aux Etats-Unis. Les sujets que j'ai utilisés provenaient de Pica pica. Le cycle complet a été suivi expérimentalement de l'adulte à l'adulte (J. Timon-David, 1960).

Cinq lots d'Hélicelles ont été contaminés au cours de plusieurs années successives, avec des taux d'infestation variant de 62 à $84 \%$. Il y a deux générations de sporocystes: la forme primaire est immobile, tandis que les sporocystes fils se déplacent activement chez le Mollusque; ils prennent naissance en grand nombre ; ils possèdent une double paroi : l'enveloppe interne (endocyste), épaisse et résistante, se colore électivement par le bleu d'aniline; elle joue un rôle essentiel dans la protection des cercaires contre la dessication lorsque les sporocystes sont rejetés par l'Hélicelle dans le milieu extérieur; chacun d'eux renferme un petit nombre (6 à 8) de xiphidiocercaires à queue courte. Ces sporocystes sont émis par le pneumostome; leur rejet est contrôlé par les facteurs écologiques, et en particulier, par l'humidité. Les cercaires qu'ils contiennent peuvent ainsi vivre 48 heures dans les conditions expérimentales.

Le cycle comporte un second hôte intermédiaire qui est un Isopode (Armadillo ou Armadillidium) dont la contamination a été réalisée. Ces Isopodes renfermant les métacercaires enkystées ont été administrés à des Moineaux friquets (Passer montanus) chez lesquels l'adulte a été retrouvé dans la vésicule biliaire.

L'émission des sporocystes a débuté 76 jours après la contamination de l'Hélicelle ; elle s'est prolongée pendant plus de trois mois dans mes expériences. La maturation des métacercaires chez les Isopodes demande 37 jours; la durée totale du cycle est relativement longue ( 9 mois).

C) Brachylecithum alfortense (A. Railliet). Ce Distome est très commun dans les canalicules biliaires de la Pie: sa fréquence varie de 45 à $75 \%$ aux environs d'Aix. Les Hélicelles ont été contaminées à trois reprises, avec des taux positifs allant de 25 à $80 \%$. Les sporocystes secondaires sont formés 65 jours après le repas 
infestant; leur nombre est immense (plusieurs milliers par Mollusque); ils mesurent à ce moment 210 à $420 \mu$; leur taille atteint 600 à $800 \mu$ au bout de 95 jours; ils commencent alors à montrer quelques mouvements lents ; leur forme est cylindrique et chacun ne renferme qu'un petit nombre de cercaires ( 3 à 5 en général).

Les sporocystes âgés de 120 jours mesurent 1,1 à $1,4 \mathrm{~mm}$; leurs mouvements sont alors très accusés: ils progressent par une sorte de reptation. Les cercaires ne se développent que lentement: leur queue ne commence à s'individualiser que vers 144 jours ; elles sont complètement développées à 240 jours. Ce sont des xiphidiocercaires à longue queue et grosse vessie épithéliale du type vitrina. Aucune forme enkystée n'a été observée.

La suite du cycle est encore inconnue. On peut supposer, par analogie avec ce qui existe chez d'autres Dicrocoeliidés, qu'un second hôte intermédiaire (peut-être un Arthropode ?) doit être nécessaire.

D) Paradistomum mutabile (Molin 1859) Travassos 1919. Ce parasite est extrêmement fréquent dans la vésicule biliaire de Lacerta muralis (Laurenti), à l'île de Riou : sur 30 Lézards disséqués, 28 ont été trouvés porteurs de Paradistomum, avec une fréquence de 2 à 20 par hôte. C'est ce matériel qui a été utilisé pour contaminer des Hélicelles provenant des environs d'Aix. Le taux de contamination a atteint $50 \%$ dans une première expérience et $20 \%$ dans une seconde ; c'est le plus faible qui ait été obtenu pour tous les Distomes étudiés dans ce travail.

Le développement est lent. Il y a deux générations de sporocystes. Les sporocystes fils, examinés 194 jours après la contamination, mesurent environ $1 \mathrm{~mm}$; chacun renferme 5 à 6 cercaires, entourées par un endocyste très net; elles ont une queue très courte, en moignon, et sont armées d'un stylet ; elles possèdent une grosse vessie à paroi épithéliale et des glandes de pénétration para- et rétro-acétabulaires.

La suite du cycle est encore inconnue: les recherches à poursuivre doivent éclaircir le mode d'expulsion des sporocystes et l'existence possible d'un second hôte intermédiaire.

\section{$3^{\circ}$ Développement de deux espèces de Leucochloridiidae.}

A) J'ai réalisé le cycle expérimental complet d'Urotocus tholonetensis T. D. L'adulte est parasite dans la bourse de Fabricius de la Pie (1955, 1957). Six lots d'Hélicelles ont été contaminés, tous avec des résultats positifs variant de 75 à $87 \%$. Par ailleurs, deux Hélicelles ont été trouvées au Tholonet avec une infestation naturelle à Urotocus.

Les sporocystes qui sont fortement ramifiés, avec des diverticules souvent renflés en massue, se développent dans la glande digestive de l'Hélicelle; ils ne montrent aucun mouvement ; leur taille, 45 jours après la contamination, varie de 0,8 à $1,2 \mathrm{~mm}$; A ce stade, ils ne renferment que des balles germinales; ils atteignent $2 \mathrm{~mm}$ au bout de 65 jours et $4,3 \mathrm{~mm}$ au bout de 113 jours; ils contiennent alors des cercaires, au nombre d'une centaine, qui sont complètement dépourvues de queue; elles s'enkystent sur place, à l'intérieur du sporocyste. Les premières métacercaires enkystées ont été observées 125 jours après le repas infestant. 
Depuis la publication de mon mémoire de 1957, j’ai réussi à boucler complètement le cycle expérimental en contaminant avec succès un jeune pigeon qui avait absorbé des Hélicelles avec métacercaires mûres. Deux Urotocus ont été obtenues dans la bourse de Fabricius ( 268 jours après la contaminaton initiale des Hélicelles).

B) Une autre série de recherches expérimentales encore en cours a porté sur Dollfusinus frontalis Biocca et Ferretti 1958, parasite des sinus frontaux du Hérisson (Erinaceus europaeus L.). Les auteurs italiens n'ayant pas précisé exactement leur opinion sur la position systématique de ce Distome, je propose de le rattacher aux Leucochloridiidae (sensu Dollfus), en raison de la situation de son pore génital qui est terminal.

J'ai retrouvé $D$. frontalis en Provence et j'ai pu obtenir à cinq reprises son développement chez $H$. arenosa, avec des taux d'infestation atteignant $86 \%$.

Les sporocystes fils mesurent 0,8 à $1 \mathrm{~mm}, 46$ jours après la contamination ; ils peuvent être facilement isolés par dissection de la glande digestive ; ils sont constitués par un sac central, ovoïde ou piriforme, d'où se détachent des diverticules d'inégale longueur qui sont très mobiles. Les sporocystes âgés de 82 jours mesurent $2,5 \mathrm{~mm}$ et contiennent de nombreuses cercaires à queue très courte qui occupent aussi bien le sac central que les diverticules; leur description détaillée est donnée ailleurs.

Il suffit de placer les Hélicelles en milieu saturé d'humidité pour observer l'émission des cercaires à l'extérieur (18 semaines après la contamination initiale). Chaque Mollusque peut ainsi émettre une centaine de cercaires par 24 heures. La voie de sortie utilise aussi bien le tube digestif que la cavité respiratoire.

La suite du développement nécessite la présence d'un second Mollusque: Euparyphia pisana (Müller). Les cercaires pénètrent facilement par le pneumostome et se rassemblent en grand nombre dans la cavité péricardique ; c'est là qu'elles grandissent et mûrissent pour se transformer en métacercaires infestantes (non enkystées).

\section{$4^{\circ}$ Développement d'un Brachylaemidae.}

J'ai déjà signalé (1953, p. 259-60) que les Hélicelles de la région du Tholonet sont très fréquemment parasitées par des métacercaires de Brachylaemus fuscatus (Rudolphi), correspondant à la forme adulte qui vit dans le tube digestif de la Pie. Ces métacercaires se trouvent, en particulier, dans le rein et dans la cavité péricardique.

En revanche, il est excessivement rare de trouver dans la nature des Hélicelles avec des sporocystes et des cercaires dans la glande digestive : on peut en examiner des centaines sans en trouver une seule, mais on peut les obtenir sans difficultés par contamination expérimentale.

Dans un lot d'Hélicelles contaminées par des œufs de B. fuscatus, le taux d'infestation a atteint $62 \%$. Les cercaires se développent assez lentement. Chez les Mollusques contaminés depuis six mois, on trouve la glande digestive profondément désorganisée par la présence de sporocystes ramifiés et très longs: j'ai pu disséquer un exemplaire dépassant $1 \mathrm{~cm}$. Les cercaires, qui ont une queue très courte, en moignon, 
sont extrêmement actives, se contractant sans arrêt. Leur morphologie est conforme aux descriptions déjà données. Les coupes les montrent fortement agrippées au sporocyste dont elles pincent la paroi avec leurs ventouses.

(Laboratoire de Zoologie générale de la Faculté des Sciences de Marseille)

\section{Ouvrages cités}

Biocca (E.) et Ferreti (G.), 1958. - Un nuovo Trematode Dollfusinus frontalis gen. nov. et sp. nov., parassita dei seni naso frontali di Erinaceus europaeus. Rendic. Accad. Naz. Lincei, 24, 171-175.

GinetsinsKaia (T. A.), 1949. - Cycle évolutif du Trématode Cyclocoelum microstomum (Creplin 1829) (en Russe). Dokl. Akad. Nauk. S.S.S.R., 66, 1219-1222.

PaLM (V.), 1963. - Der Entwicklungszyklus von Transcoelum oculeus (Kossack 1911) Witenberg 1923. (Fam. Cyclocoelidae) aus dem Blesshuhn (Fulica atra L.). Zeit. für Parasitenk., 22, 560-567.

StUNKaRd (H. W.), 1923. - The life cycle of Typhlocoelum cymbium. Journ. of Parasitol., 20, 336.

Szidat (L.), 1923. - Zur Entwicklungsgeschichte der Cyclocoeliden. Der Lebenszyclus von Tracheophilus sisowi Skrj. Zool. Anz., 100, 205-213.

Timon-David (J.), 1953. - Recherches sur les Trématodes de la Pie en Provence. Ann. Parasitol. Hum. et Comp., 27, 247-288.

—, 1955. - Cycle évolutif d'un Trématode Cyclocoelidé, Pseudhyptiasmus dollfusi T.D. Recherches expérimentales. Ibid., 30, 43-61.

-, 1957. - Recherches expérimentales sur le cycle évolutif d'un Trématode du genre Urotocus Looss (Digenea, Leucochloridiidae). Ann. Parasitol. Hum. et Comp., 32, 219-242.

— ,1957. - Nouvelles recherches expérimentales sur le cycle évolutif du Trématode Pseudhyptiasmus dollfusi T. D. (Digenea, Cyclocoelidae). C.R. Soc. Biol., 151, 592-594.

-, 1957. - Recherches sur le développement expérimental de Brachylecithum alfortense (A. Railliet), R.-Ph. Dollfus 1954, Trématode Dicrocoeliidé parasite des voies biliaires de la Pie. Ann. Parasitol. Hum. et Comp., 32, 353-368.

—, 1960. - Recherches expérimentales sur le cycle de Dicrocoelioides petiolatum (A. Railliet 1900) (Trematoda, Dicrocoeliidae. Ibid., 35, 251-267.

-, 1964. - Développement expérimental et formes larvaires de Dollfusinus frontalis Biocca et Ferretti 1958 (Trematoda, Digenea), parasite des sinus frontaux du Hérisson. C.R. Ac. Sc., 258, 3755-3757.

-, 1964. - Contribution à la connaissance de la spécificité parasitaire chez quelques Trématodes Digènes évoluant chez des Mollusques Pulmonés terrestres. Premier Congrès International de Parasitol., Rome (sous presse).

Williams (I. C.), 1960. - The anatomy and some aspects of the biology of Urotocus rossittensis (Mühling 1898) Looss 1899 (Trematoda : Brachylaemidae) from the bursa Fabricii of the Rock-Pipit Anthus spinoletta petrosus (Montagu). Ann. and Mag. Nat. Hist., $13,65-86$.

Wright (C. A.), 1960. - Relationships between Trematodes and Molluscs. Ann. Trop. Med. Parasitol., 54, 1-7. 